\title{
Diversidad de la entomofauna asociada a vegetación aledaña a cultivos de arroz, maíz y algodón
}

\section{Entomofauna diversity associated to sorrounding vegetation on rice, corn and cotton crops}

\author{
Adolfo Bedoya ${ }^{1}$, Claudio Fernández Herrera², Karol Darío Pérez García ${ }^{3 *}$ \\ Recibido para publicación: Septiembre 5 de 2017 - Aceptado para publicación: Mayo 24 de 2018
}

\begin{abstract}
RESUMEN
Las arvenses aledañas a agroecosistemas ofrecen respuestas al comportamiento de muchas especies fitófagas, depredadoras y parasitoides. Para ello se determinó la diversidad de organismos entomófagos y fitófagos en la vegetación aledaña a cultivos de arroz, maíz y algodón. Se establecieron estaciones de muestreo distribuidas al azar y se determinaron las especies vegetales. La identificación de arvenses llego a nivel de especie; la entomofauna se colectó con red entomológica, pitfall y en forma manual. El análisis de diversidad de artrópodos se realizó utilizando el método propuesto por Jost, para el grado de recambio se calculó a través de tres valores $q=0,1$ y 2 y se construyeron curvas de rango-abundancia. Las especies vegetales se agruparon en 16 familias y 21 géneros; siendo Ruellia tuberosa y Euphorbia hirta hospederas de enemigos naturales, mientras que Heliotropium indicum, Caperonia palustris y Amaranthus dubius fueron altamente hospedante de fitófagos. El análisis de completitud a partir de la cobertura de la muestra de cada borde de cultivo y los roles tróficos fitófago, parasitoide y depredador presentaron una completitud mayor a 99\%. La comparación de medias mostró diferencias significativas de la fauna asociada entre los bordes de cultivo Arroz-Maíz $(Z=2,6929 ; \quad p=0,0212)$ y ArrozAlgodón ( $Z=3,7634 ; p=0,0005)$, sin embargo, los bordes Maíz-Algodón no mostraron diferencias significativas $(Z=1,0705 ; p=0,8532)$. Se puede concluir que el borde de los agroecosistemas maíz, arroz y algodón presentan atributos estructurales que influyen significativamente en la dinámica y diversidad de la entomofauna asociada.
\end{abstract}

Palabras clave: Arvenses, agroecosistema, biodiversidad, fitófagos, benéficos.

\begin{abstract}
The surrounding weeds to agro-ecosystems provide answers to the behavior of many phytophagous species, besides predators and parasitoids. In order to find out that the diversity of organisms entomophagous was determined and surrounding vegetation to rice, corn and cotton were established. Sampling stations were randomly distributed and plant species were determined. The weed identification was performed at species level; the entomofauna was collected with jama, pitfall and manually. The arthropod diversity analysis was performed using the method proposed by Jost; the degree of replacement method was calculated through three values $q=0,1$ and 2 and range-abundance curves were constructed. Plant species were grouped into 16 families and 21 genera; being uellia tuberosa and Euphorbia hirta host of natural enemies, while Heliotropium indicum, Caperonia palustris y Amaranthus dubius were hosts of high quantities of phytophagous. The completeness analysis from sample coverage of each crop edge and the trophic roles: phytophagous, parasitoid and predator had a higher than 99\% completeness. Comparison of means showed significant differences for associated fauna between the edges of rice-corn crops $Z=2.6929$ $p=0,0212)$ and rice-cotton $(Z=3.7634 p=0,0005)$ however, the corn-cotton edges showed no significant difference $(Z=1.0705 p=0.8532)$. It can be concluded that the agroecosystem edge of maize, rice and cotton present structural attributes that significantly influence the dynamics and diversity of the associated entomofauna.
\end{abstract}

Key words: Weed, agricultural ecosystem, biodiversity, phytophagous, beneficial.

\footnotetext{
'Ing. Agrónomo, Universidad de Córdoba. Montería, Córdoba, Colombia Tel. (571) 3830444 Ext 1124, Fax: (571) 3830444 Ext $1200 . * A u t o r$ para correspondencia: adolfobedoya@gmail.com

${ }^{2}$ Ing. Agrónomo, Docente. Facultad de Ciencias Agrícolas, Universidad de Córdoba. E-mail: claudiofernandezherrera@gmail.com

3* Biólogo, Docente. Facultad de Ciencias Agrícolas. Universidad de Córdoba. Montería, Córdoba, Colombia. A. A. 354. Tel. +57(4)

7860255 E-mail:karolperez0414@hotmail.com
} 


\section{INTRODUCCIÓN}

El acelerado aumento de la frontera agrícola cordobesa trajo consigo la transformación de la región en zonas de vegetación ecológicamente homogéneas, promoviendo con esto la reducción y posterior desaparición de parches de vegetación natural que contribuyen al sostenimiento de un sin número de especies entomófagas de gran importancia en programas de manejo integrado de plagas (Marshall y Moonen, 2002).

El uso de productos para el mantenimiento del cultivo, conlleva a la captación de bajos ingresos por parte de los productores. A nivel de salud pública existen riesgos de desarrollo de enfermedades o trastornos a largo plazo en la comunidad consumidora (Rodríguez y Van Hoof, 2003). Tal vez una de las peores consecuencias de la homogenización de la vegetación, la constituye el desequilibrio de la biodiversidad (Altieri y Nicholls, 2012).

Una tendencia desafortunada que acompaña a la expansión de los monocultivos, es que ésta ocurre a expensas de la vegetación natural circundante que sirve para mantener la biodiversidad a nivel del paisaje (Altieri y Nicholls, 2002). La importancia de la vegetación adyacente conlleva al interés por el control biológico natural y el conocimiento de la dinámica de población de las plagas y de sus enemigos naturales (Marshall y Moonen, 2002).

Los estudios se han enfocado primordialmente en el efecto de vegetación nativa, sin embargo los cultivos de tipo perenne también pueden influir de manera positiva. Las arvenses pueden contribuir a la creación de un ecosistema con características cercanas a las de un bosque secundario (Rodríguez y Van Hoof, 2003). Las funciones que brindan los márgenes son diversas, sin embargo su función como hábitat ha sido esclarecida en los últimos años; Denys y Tscharntke (2002) realizaron una recopilación de investigaciones en torno al tema donde se han evidenciado los servicios ambientales que brindan los márgenes a los cultivos.

Adicionalmente, estudios han mostrado que depredadores polífagos presentan mayores densidades cerca de los márgenes (15-30 m), aumentando de esta manera el control de plagas en las hileras de cultivo cercanas a bordes (Altieri y Nicholls, 2012). Estudios realizados en parches no cultivados adyacentes a cultivos determinaron que zonas en barbecho y amplias franjas no cultivadas pueden otorgar mayores ventajas en el control de plagas que márgenes delgados (Denys y Tscharntke, 2002). Según Nicholls (2006) una consecuencia de esta tendencia es que la cantidad total de hábitat disponible está decreciendo a tasas alarmantes.

La importancia de los corredores naturales en algunos tipos de cultivos ha resultado determinante en lo que se refiere al control de plagas: Nicholls et al. (2001) evaluaron el efecto de vegetación riparia sobre la distribución y abundancia de las plagas Erythroneura elegantula Osborn (Hemiptera: Cicadellidae), Frankliniella occidentalis (Pergande) (Thysanoptera: Thripidae) y depredadores generalistas en viñedos.

Este tipo de estudios han ido demostrando que la eficacia de los márgenes para un posible control biológico ya que permiten a poblaciones de niveles tróficos altos mantenerse a través de los años; no obstante, Nicholls (2008) afirma que el control biológico de plagas dejó de practicarse con la generalización de la lucha química como medida de control de plagas, enfermedades y arvenses hasta que, por los diversos problemas que ocasionó el uso intensivo de plaguicidas, ganó de nuevo terreno como alternativa en el manejo de la salud vegetal. Los márgenes de cultivos también pueden brindar beneficios como barreras de dispersión y movimiento de plagas (Altieri y Nicholls, 2012). También ofrecen alternativas que permitan a los 
productores reducir los costos de producción a mediano y largo plazo, al incorporar una visión a mayor escala que tenga en cuenta los procesos que suceden en el paisaje (Bennett et al., 2006).

Todos los agroecosistemas son dinámicos y están sujetos a diferentes tipos de manejo, de manera que los arreglos de cultivos en el tiempo y en el espacio están cambiando continuamente de acuerdo con factores biológicos, socioeconómicos y ambientales (Nicholls, 2004). Es por ello que en Córdoba y en especial en el municipio de Montería el conocimiento la diversidad de organismos entomófagos y vegetación aledaña a cultivos de arroz (Oryza sativa), maíz (Zea mays), y algodón (Gossypium hirsutum), es de gran importancia para establecer mecanismos que ofrezcan respuestas al comportamiento de muchas de estas especies fitófagas, depredadoras y parasitoides fuera de los agroecosistemas.

\section{MATERIALES Y MÉTODOS}

El estudio se realizó en el municipio de Montería ubicado en la parte norte del medio Sinú entre los meridianos $8^{\circ} 45^{\prime} 27^{\prime \prime}$ latitud norte y $75^{\circ}$ $53^{\prime} 24^{\prime \prime}$ longitud oeste, las unidades espaciales de análisis desarrollados en la investigación corresponden a zonas contiguas a cultivos de arroz, algodón y maíz localizados en la zona norte y noreste de la ciudad de Montería.

Para realizar el estudio se estableció un premuestreo alrededor de los agroecosistemas establecidos dentro la Universidad de Córdoba donde se determinaron los tipos de especies vegetales y organismos entomófagos presentes, para diseñar junto con las bibliografías de las especies ya registradas en la zona, una hoja de campo donde se referenció un total aproximado de cuáles son las especies de vegetación y entomofauna existentes.

Las especies de vegetación y organismos entomófagos que no fueron referenciados se colectaron para su posterior identificación taxonómica. La identificación de las arvenses se hizo hasta el nivel de especie, algunas fueron colectadas, preparadas e identificadas en el herbario de la Universidad de Córdoba, por comparación de especímenes y/o utilizando literatura y claves de varios autores como Liogier (1982, 1983, 1985, 1986, 1989, 1994, 1995, 1996 y 2000), Byrd (1978) y Bailey y Bailey (1976). También se realizó monitoreo mediante observaciones directas para identificar la entomofauna benéfica y fitófaga y la interacción insecto-planta.

La fase de campo, se realizó en un período de doce meses aproximadamente donde se tomó lectura en las dos épocas del año presentes en la zona (época seca y época de lluvia). Se establecieron estaciones de muestreo fijas dispuestas en la vegetación natural fuera de cada cultivo, de diez metros de largo por tres de ancho.

Se procedió a realizar diez pases doble de jama colectando todos los insectos voladores asociados a estos parches de vegetación natural, también se instalaron trampas Pitfall utilizando vasos plásticos de 3, 5 y 12 onzas con el objetivo de muestrear de artrópodos del suelo, para esto se ubicaron diez (10) puntos sobre los bordes de los agroecosistemas.

Para la captura de los agentes entomófagos forrajeadores asociados en la vegetación natural se hizo de forma manual donde se capturó el ejemplar y posteriormente se estableció su interacción o la actividad que este presentaba en el medio. Los organismos se almacenaban en bolsas de papel kraft, con el fin que se facilitara la absorción de humedad de la artropofauna y se sacrificaron en el laboratorio de entomología de la Universidad de Córdoba por medio de la técnica de refrigeración.

Durante la fase de laboratorio se separó el material colectado a morfoespecies o a unidades taxonómicas reconocibles y se 
conformó una colección de referencia que se encuentra disponible en el laboratorio de entomología del programa de Ingeniería Agronómica de la Universidad de Córdoba.

Los ejemplares analizados en el laboratorio fueron identificado hasta la categoría taxonómica más específica posible, con ayuda del estereoscopio (Advanced optical JSZ6S con cámara incorporada INFINITY 1) y utilizando claves taxonómicas de Fernández and Sharkey (2006); McGavin (2000, 2001); Borror y White (1998); Triplehorn y Johnson (2011) y McAlpine et al. (1981). El material biológico fue preservado en seco de acuerdo a la naturaleza de los ejemplares.

Para el análisis de la diversidad de artrópodos se realizó utilizando el método propuesto por Jost (2006) mediante los números efectivos de especies, este análisis permite evaluar directamente la magnitud de cambio entre las comunidades estudiadas y expresarla en términos del número real de especies.

La diversidad y el grado de recambio de especies reales se calculó a través de tres valores de $q=0,1$ y 2, según Chao y Jost (2012). El valor $q=0$ es la diversidad de orden cero $\left({ }^{\circ} \mathrm{D}\right)$, donde no se consideran las abundancias de las especies, por lo que equivale a la riqueza de especies, $q=1$ es la diversidad $\left({ }^{1} \mathrm{D}\right)$, donde todas las especies son incluidas con un peso proporcional a su abundancia en el ensamblaje, siendo el índice de diversidad ( $\left.{ }^{1} D\right)$ el exponencial del índice de entropía de Shannon y $q=2$ es la diversidad $\left({ }^{2} \mathrm{D}\right)$, que es el inverso del índice de Simpson, que tiene en cuenta a las especies dominantes y excluye a las raras según Jost (2007) y Moreno et al. (2011).

La completitud del muestreo se estimó mediante el análisis de coberturas estandarizadas de las muestras $(I C=95 \%)$. Este valor fue calculado restando el límite superior de cobertura (UCL) menos el número de especies calculadas $(\mathrm{Sm})$; se generaron intervalos de confianza al 95\%. Se construyeron curvas de rango-abundancia o curvas de Whittaker según Magurran (2004) y Feinsinger (2003), en las que se usó el número de especies y de individuos por especie registrados en cada tipo de cultivo. La curva se graficó de acuerdo con el logaritmo de las abundancias observadas y los datos se ordenaron desde la especie más abundante a la menos abundante.

Se realizaron pruebas de comparación de muestras independientes (T-student y MannWhitney $U$ ) para verificar si existía una diferencia entre la diversidad de especies y la vegetación aledaña a los tipos de cultivos.

\section{RESULTADOS Y DISCUSIÓN}

Las especies vegetales que proveen de recursos alimenticios a los artrópodos se agruparon en 24 especies las cuales eran plantas arvenses florecidas, discriminadas en 16 familias y 21 géneros, representadas con el 4,16\% para las monocotiledóneas y el 95,84\% para las dicotiledóneas. Las familias Malvaceae y Fabaceae fueron las que presentaron mayor riqueza con tres géneros y tres especies, seguidas por Euphorbiaceae con dos géneros y tres especies, Convolvulaceae y Boraginaceae con un género y dos especies cada una. Las familias Acanthaceae, Amaranthaceae, Asteraceae, Commelinaceae, Onagraceae, Poaceae, Nyctaginaceae, Myrtaceae, Passifloraceae, Portulacaceae y Zygophyllaceae estuvieron representadas por un género y una especie.

Estos resultados confirman que todavía existen corredores y parches de vegetación natural que actúan como reservorio de semillas o propágulos para evitar la pérdida de las especies espontáneas. De las 24 especies descritas solo siete (7) fueron perennes a pesar de que se presentó un alto número de especies dicotiledóneas, teniendo el resto un ciclo de vida anual. La alta población de especies vegetales de ciclo corto en los 
agroecosistemas puede estar asociada a los precedentes culturales, entre ellos el tipo cultivo (arroz, maíz y algodón), las labores de manejo mecánico y químico empleadas para su control, así como la composición del banco de semillas como se dijo anteriormente presente en el suelo y las condiciones ambientales que pudieron haber influido en su germinación y distribución de forma espontánea.

En las observaciones realizadas la mayoría de las plantas arvenses permanecieron en estado de floración, notándose una alta densidad de especies polinizadoras, entomófagas, fitófagas, depredadoras y parasitoides debido a la presencia de polen y de néctar en las flores, sirviendo como fuente de alimentación alterna para estos insectos. Resultados que se asemejan a los reportados Landis et al. (2000) quienes afirman que estos hábitats son importantes como sitios de refugio ya que proveen alimento para enemigos naturales en épocas de escasez de plagas en los sistemas productivos, además del polen y el néctar.

Observación directa de las arvenses y entomofauna benéfica. Dentro de los resultados se pudo establecer que muchas de estas plantas arvenses hospedaron muchos insectos benéficos, tal es el caso para los parasitoides donde la arvense Ruellia tuberosa albergó una alta presencia de insectos de la familia Eulophidae (54,2\%) especialmente la especie Euplectrus sp. y dos morfoespecies del total de las capturas realizadas. De igual forma durante el conteo visual realizado en campo mostró el mayor número de visitas. Seguidamente, Euphorbia hirta y Tridax procumbens se presentaron como las plantas hospederas de segunda importancia con el 12,4\%.

Estos resultados concuerdan con los reportados por Saini y Polack (2002) quienes afirmaron que especies de la familia Eulophidae son reconocidas a nivel mundial como agentes de biocontrol de trips, y en países tales como Holanda, Bélgica, Italia, Francia, Alemania, Estados Unidos y Canadá, son comercializados como insumos biológicos.

De igual forma Paleologos et al. (2008); describieron en este sentido, la presencia de especies pertenecientes a las familias Apiáceas, Asteráceas y Fabáceas en cultivos hortícolas, estaría indicando la existencia de hábitats de enemigos naturales especialmente de la familia Eulophidae, siendo potenciales parasitoides de algunas de las plagas más comunes. Esto demuestra la existencia de un potencial control natural de regulación y puede ser un importante recurso para aquellos agricultores que no pueden o no desean adquirir insumos químicos para el control de las plagas.

Por su parte la familia Braconidae tuvo un alto número de plantas hospederas entre las que se destacan Ruellia tuberosa y Euphorbia hirta con un 10,3\% cada una; evidenciándose principalmente las especies Bracon sp., Apanteles sp. y Chelonus sp., por su parte las arvenses Ipomoea trifida y Desmodium tortuosum hospedaron solo el 5,1\%. Para la familia Chalcididae las arvenses que albergaron el mayor número de insectos fueron Gliricidia sepium, Euphorbia hirta, Ipomoea trifida y Echinochloa colona respectivamente.

En todas estas arvenses se destaca la presencia de las especies de insectos como Conura sp. y Brachymeria sp.

De la familia Ichneumonidae, las arvenses a las cuales se les encontró presencia de estos insectos fue Ipomoea trifida y Gliricidia sepium siendo esta última a la cual se observó directamente el mayor número de insectos en campo tales como la especie Casinaria sp. 
Los insectos de la familia Pteromalidae se observaron en un $25 \%$ de las arvenses, presentándose el mayor número de individuos en la especie Euphorbia hirta con el $26,5 \%$ del total de insectos colectados, Tridax procumbens y Gliricidia sepium con el 14,7\% e Ipomoea trifida con $11,8 \%$ respectivamente.

Las familias de insectos que solo se evidenciaron hospedadas en una sola planta arvense fueron: Dryinidae con una sola morfoespecie quien se observó interactuando en la arvense Heliotropium curassavicum; la familia Phoridae con dos morfoespecies en la arvense Echinochloa colona; la familia Signiphoridae con dos morfoespecies en la arvense Sida acuta y por último la familia Torymidae con una morfoespecie en la arvense Euphorbia hirta. Estos insectos se dedicaban a posarse sobre las flores de las arvenses con la finalidad de alimentarse de los recursos que éstas le proveían.

La familia Tachinidae se observó refugiándose en siete plantas entre las que se destacan Heliotropium curassavicum $(40,6 \%)$ y Euphorbia hirta (34,3\%) donde se alimentaban del néctar de las flores y en algunos casos se hallaban forrajeando toda la planta; se destacan la especie Architas sp. y una morfoespecie.

Para los insectos depredadores la familia Vespidae fue la que mayor numero presentó sobre las arvenses como Boerhaavia erecta con un 20,8\% además de Heliotropium curassavicum, Ipomoea trifida, Sida acuta y Kallstroemia maxima que presentaron el $12,5 \%$ cada una. Estas especies vegetales proveen de alimentos como polen y néctar a las avispas de esta familia las cuales al no encontrar sus presas tratan de compensar su dieta con este tipo de recursos, estas especies de insectos estaban representadas por Polybia occidentalis y Agelaia myrmecophila.

La familia Formicidae tuvo una alta participación, encontrándose presente en el $54,2 \%$ de todas las especies vegetales evaluadas en el estudio. Las plantas que hospedaron el mayor número de ejemplares fueron Euphorbia hirta la cual hospedó el mayor número de especies de insectos tales como Brachimirmex sp., Solenopsis sp., Pheidole sp., Dorymyrmex sp. y Paratrechina sp.; seguida por la arvense Myrcianthes sp. la cual hospedó especies de insectos como Brachimirmex sp., Solenopsis sp., Pheidole sp.; y por último la arvense que Ipomoea trífida la cual albergó a las especies Dorymyrmex sp., Paratrechina sp. y Pseudomirmex sp.; por su parte las arvenses Malachra fasciata, Anoda acerifolia, Desmodium tortuosum, Kallstroemia maxima y Sida acuta estuvieron representadas por las especies Crematogaster sp., Ectatoma sp1., Ectatoma sp2., Odontomachus sp. respectivamente, los cuales no superaban el $8 \%$ del total de especies asociadas a las arvenses.

La familia Anthocoridae se presentó únicamente en las arvenses Ruellia tuberosa, Ipomoea trifida y Tridax procumbens respectivamente con las especies Orius sp.1 y Orius sp. 2. Por otra parte, del total de insectos colectados de la familia Dolichopodidae, se pudo observar que hubo una mayor presencia en la arvense Gliricidia sepium (35\%), Amaranthus dubius (20\%) e Ipomoea trifida (15\%); todas ellas hospedando a la especie Condylostylus sp. El resto de las arvenses no superaban el $10 \%$ de asocio con esta especie de depredador. Estos resultados nos dan una clara visión de los principales reservorio de arvenses para mantener en 
campo a este importantísimo benéfico.

Las arvenses que hospedaron la mayor parte de los insectos de la familia Syrphidae fueron Boerhaavia erecta, Commelina diffusa, Amaranthus dubius y Echinochloa colona, donde principalmente predominaban las especies Allograpta sp., Baccha sp. y Mesograpta sp. Se pudo notar que éstas especies generalmente se encontraban alimentándose del néctar y polen de las flores. El género Mesograpta sp. fue el más abundante en el estudio, lo cual demuestra la importancia que este insecto puede representar en el control biológico natural de muchos insectos fitófagos presentes en las áreas de cultivos.

La familia Coccinellidae se observó en un alto número en las arvenses Amaranthus dubius, Ipomoea trifida, Gliricidia sepium, Ludwigia hyssopifolia y Malachra fasciata. El género Coleomegilla fue el más representativo, donde los adultos se encontraban forrajeando en la toda la planta. Esta familia juega un papel muy importante en el control biológico en nuestra región ya que se alimenta de áfidos, ácaros y huevos de insectos entre ellos los de Spodoptera frugiperda que se constituye en una limitante para muchos cultivos. También se encontraron las especies Cicloneda sanguínea, Olla sp., Scymnus sp., Stethorus sp. e Hyperaspis sp. pero en una menor proporción en comparación a la especie Coleomegilla maculata.

La familia Reduviidae estuvo representada con las especies Pselliopus sp., Triatoma sp. y Zellus sp.; de igual manera la familia Therevidae con una morfoespecie presentándose ambas en la arvense Ipomoea trífida; además de lo anterior, Pselliopus sp., Triatoma sp. y Zellus sp. también se asociaron a la arvense Ruellia tuberosa la familia Reduviidae y Therevidae a la arvense Euphorbia hirta, resaltando que ambas familias se encontraban alimentándose de polen y néctar de las flores. Estos eventos se asemejan a los expresados por Norris y Kogan (2005); Nicholls (2008); Gamboa y Criollo (2011), quienes afirman los insectos benéficos encuentran hábitats alternos en aquellos que presentan las condiciones óptimas para alimentarse y reproducirse, además de brindarles refugio.

\section{Observación directa de las arvenses e insectos fitófagos. Las observaciones} realizadas en campo reflejaron un 5\% de crisomélidos del genero Epitrix alimentándose de las hojas de la arvense Heliotropium indicum y Caperonia palustris provocando daños sobre el tejido foliar que consisten en perforaciones en forma redondeada; también, se observó en un menor porcentaje (2\%) a la especie de curculiónido Linogeraeus capillatus en los meses de febrero y abril. Estos resultados concuerdan con los reportados por Pérez et al. (2011) quienes establecieron que durante la temporada de maíz, la mayor concentración de adultos se presentó desde los bordes hasta el interior del cultivo.

La arvense Heliotropium curassavicum presenta un parentesco con la especie Heliotropium indicum, donde se observaron cantáridos del género Asclera y la especie Alkindus atratus de la familia Cydnidae de forma temporal. De igual forma la arvense Commelina diffusa hospeda también el género Asclera además de crisomélidos pertenecientes a las especies Epitrix sp. y Diabrotica balteata los cuales producían daños en hojas y la especie Neolema sp. que realizaba ataques en las flores impidiendo su desarrollo y fructificación.

Blanco y Leiva (2007; 2009; 2010), 
reportaron que la arvense Amaranthus dubius es una planta hospedera de Spodoptera frugiperda, pero cabe resaltar que en este trabajo no se evidenció la presencia de larvas ni de insectos adultos, pero sí de crisomélidos pertenecientes a los géneros Diabrotica y Disonycha los cuales producían perforaciones redondeadas en el follaje que en algunos casos provocaba la total defoliación de la planta. La arvense Euphorbia hirta, tuvo una alta población de insectos de la familia Lygaeidae; estos resultados coinciden en cierta parte con los obtenidos en Costa Rica por Mexzón y Chinchilla (2003), quienes señalaron que los principales grupos de insectos visitantes de esta planta son chinches de esta familia.

Por último podemos resaltar que los insectos polinizadores actuando sobre las arvenses Ipomoea tiliacea y Kallstroemia maxima presentaron una alta diversidad en la que se destacaron Apis mellifera y Agapostemon sp., estos resultados se asemejan a los reportados por Faye et al. (2002) y Faye y Molinelli (2004), donde muchas de las especies encontradas han sido citadas como fuente potencial de polen para las abejas (Apis mellifera) en el sudeste de Córdoba-Argentina.

Diversidad de la artropofauna asociada. Se recolectaron 28.091 individuos durante la fase de muestreo, en total se registraron 248 taxones agrupados en 126 especies y 122 a nivel de familia. Para el borde del cultivo algodón se registró el mayor número de individuos con 11.132 (39,6\%), seguido por el borde del cultivo arroz con 9.266 individuos (33\%) y por último el borde del cultivo maíz registrando 7.693 (27,4\%).

Entre los ordenes más abundantes se encuentra el orden Hemiptera representando el mayor número de individuos con 11.141 siendo el 39,7\%, seguido por el Orden Coleoptera con 6.002 individuos para un 21,4\%; luego el orden Diptera con 3.327 lo que equivale al $11,8 \%$; el orden Hymenoptera registro 3.222 individuos para un $11,5 \%$ y finalmente el orden Araneae con 1.564 individuos lo que equivale al 5,6\%, los demás ordenes no superaron el $4 \%$ del total de las muestras, siendo el orden Strepsiptera el que menor abundancia presentó con tan solo el $0,1 \%$.

\section{Comparación de la diversidad estimada de} la artropofauna. De acuerdo al análisis de completitud del muestreo estimado a partir de la cobertura de la muestra para cada área de muestreo; en este caso para cada tipo de borde de cultivo y para los roles tróficos fitófago, parasitoide y depredador, mostró que para los tres bordes de cultivos se obtuvo una completitud mayor a 99\%. Para el borde del cultivo de arroz la cobertura de la muestra fue de 0,9997 (Déficit de cobertura; $\mathrm{DC}=2,8395$ ), para el borde del cultivo de Maíz fue de 0,9990 (DC=3,8267) y por último para el borde del cultivo de Algodón de 0,9986 (DC=6,9247) (Figura 1).

Con respecto a los roles tróficos, el análisis de completitud del muestreo en los diferentes bordes de cultivos obtuvo una completitud mayor a $60 \%$. Donde el rol fitófago para el borde de cultivo Arroz la cobertura de la muestra fue de 0,6034 ( $D C=0,5587$ ), para Maíz la cobertura fue de 0,6103 (DC $=0,5324)$ y para Algodón de 0,7422 ( $\mathrm{DC}=0,4677)$.

Para el rol de parasitoide se obtuvo que para el borde del cultivo arroz una cobertura de la muestra de $0,8380(D C=1,0845)$, para Maíz un valor de 0,8454 ( $D C=1,2288)$ y para Algodón de 0,8666 ( $\mathrm{DC}=1,2092)$.

Para el rol de depredadores en Arroz mostró 


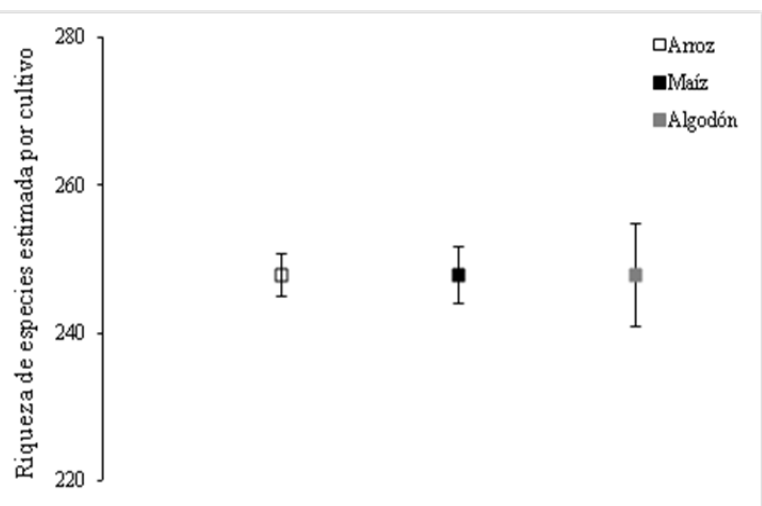

una cobertura de la muestra de 0,7187 (DC =0,5462), para Maíz fue de 0,7382 (DC $=0,6151)$ y por ultimo para Algodón un valor de 0,7268 (DC =0,7902) (Figura 2).

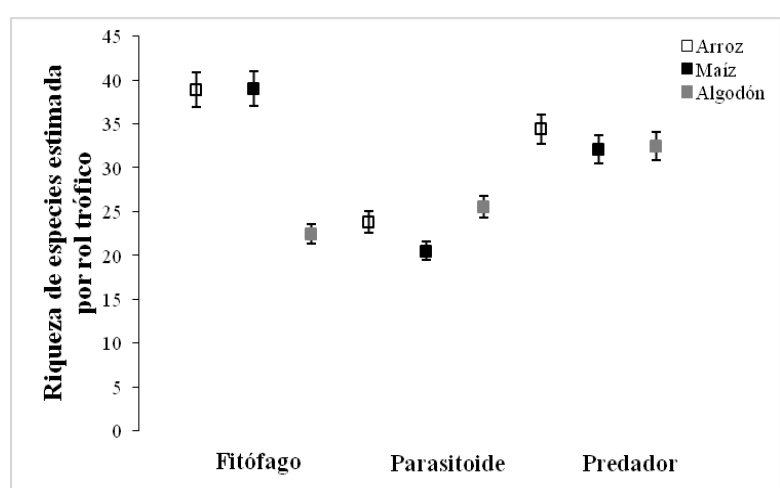

Figura 2. Riqueza de especies estimada por rol trofico asociado a los tipos de bordes de cultivos en Montería, Córdoba-Colombia.

El análisis de diversidad para los bordes de cultivos maíz y arroz presentó la misma tendencia al ser analizada la riqueza por medio de la diversidad alfa verdadera tipo ${ }^{1} \mathrm{D}$, presentada por la riqueza de especies $\left({ }^{0} \mathrm{D}\right)$, con un mayor número de especies efectivas en el borde de cultivo arroz ( $\left.{ }^{1} \mathrm{D}=139,69\right)$ comparado con el borde del cultivo maíz ( $\left.{ }^{1} \mathrm{D}=130,32\right)$; caso contrario expresó el borde de cultivo algodón ( $\left.{ }^{1} \mathrm{D}=31,81\right)$ lo que indica que las comunidades difirieron en su diversidad.

Teniendo en cuenta los valores obtenidos en los diferentes órdenes de diversidad, para el orden ${ }^{2} \mathrm{D}$ se obtuvieron valores de $(87,04)$ para el borde de cultivo arroz y de $(83,58)$ para el borde de cultivo maíz y de $(5,26)$ para el borde algodón; con base a estos últimos resultados se evidenciaron diferencias con relación a los tres perfiles de diversidad entre los agroecosistemas (Figura 3a). La diversidad de insectos entre los bordes de cultivos fueron diferentes de acuerdo a la comparación de la prueba estadística de Kruskal-Wallis (Kruskal-Wallis test: $\mathrm{H}$ $(2, N=744)=15,0522 ; p=0,0005)$, la comparación de medias mostró diferencias significativas de la fauna asociada entre los bordes de cultivo Arroz - Maíz ( $Z=2,6929$; $\mathrm{p}=0,0212)$ y Arroz - Algodón $(Z=3,7634$; $p=0,0005)$, sin embargo, los bordes Maíz - Algodón no mostraron diferencias significativas $(Z=1,0705 ; p=0,8532)$.

El análisis de diversidad alfa para el rol trófico en los cultivos de maíz, arroz y algodón no presentó la misma tendencia al ser analizada la riqueza por medio de la diversidad alfa verdadera tipo ${ }^{1} \mathrm{D}$, presentada por la riqueza de especies $\left({ }^{\circ} \mathrm{D}\right)$, con un mayor número de especies efectivas en los fitófagos ( $\left.{ }^{1} \mathrm{D}=66,59\right)$, para los parasitoides ( $\left.{ }^{1} \mathrm{D}=20,72\right)$; y para los depredadores ( $\left.{ }^{1} \mathrm{D}=48,32\right)$ lo que indica que las comunidades difirieron en su diversidad.

Los valores obtenidos en los diferentes órdenes de diversidad, para el orden ${ }^{2} \mathrm{D}$ se registraron valores teniendo en cuenta el rol funcional, para el orden ${ }^{2} \mathrm{D}$ se obtuvieron valores de $(38,37)$ para los fitófagos; de $(12,27)$ para los parasitoides y de $(37,56)$ para los depredadores con base a estos se evidenciaron diferencias con relación al perfil de diversidad de los parasitoides (Figura 3b). La prueba estadística KruskalWallis para los roles tróficos Fitófago y Depredador mostró diferencias $\mathrm{s}$ ignificativas entre la diversidad de los diferentes tipos de borde de cultivo (Kruskal-Wallis test: $\mathrm{H}(2, \mathrm{~N}=366)$ 


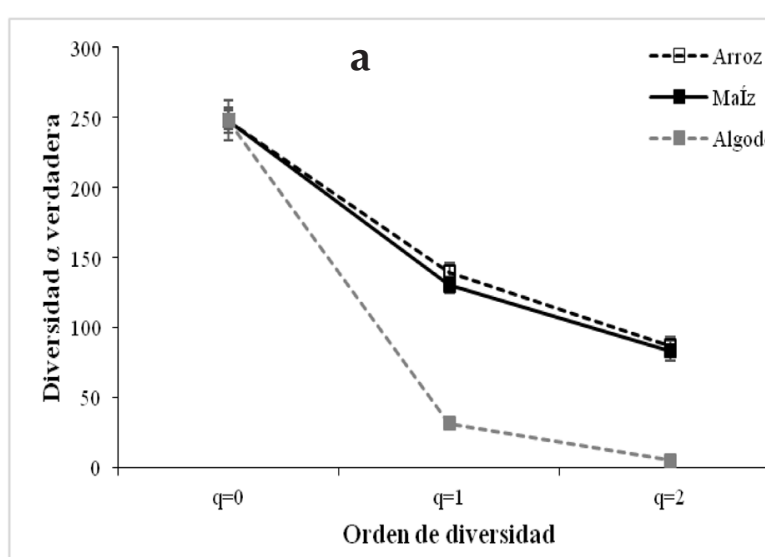

Figura 3. Perfiles de diversidad alfa verdadera (a) para los artrópodos presentes y rol funcional (b) en bordes de cultivo en Montería, Córdoba- Colombia.

$=6,5299 ; \mathrm{p}=0,0382)$ y (KruskalWallis test: $\mathrm{H}(2, \mathrm{~N}=228)=12,7693 ; \mathrm{p}$ $=0,0017$ ) respectivamente, mientras que para el rol trófico Parasitoide no mostró diferencias significativas (Kruskal-Wallis test: $H(2, N=105)=3,4205 ; p=0,1808)$.

La comparación de medias indicó que no existieron diferencias significativas entre los bordes de cultivo para los roles Fitófago y Depredador, mientras que para el rol Depredador solo los bordes Arroz - Algodón mostraron diferencias significativas $(Z=3,5180 ; p=0,0013)$. Teniendo en cuenta el grado de cambio o reemplazo en la composición de

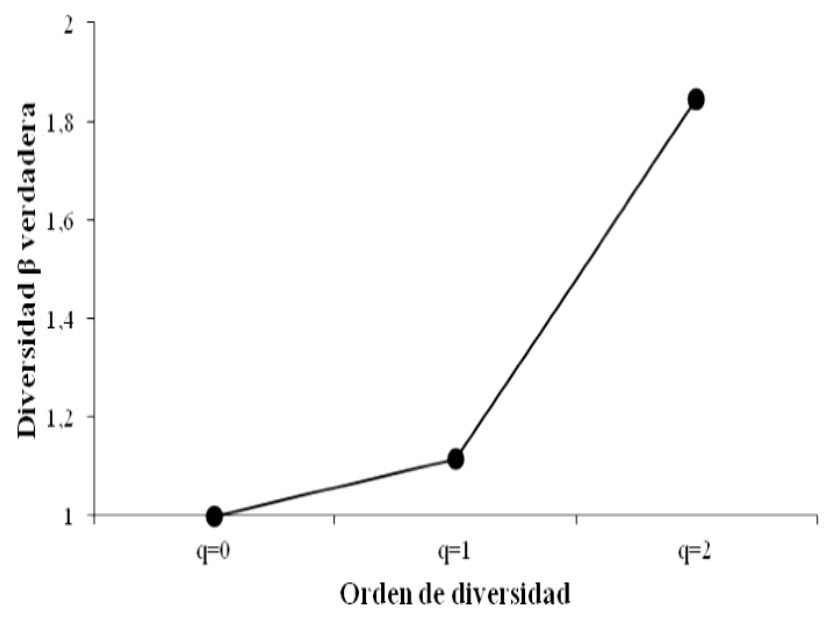

Figura 4. Perfiles de diversidad beta verdadera tes y rol funcional (b) en bordes de cultivo en Montería, Córdoba- Colombia.

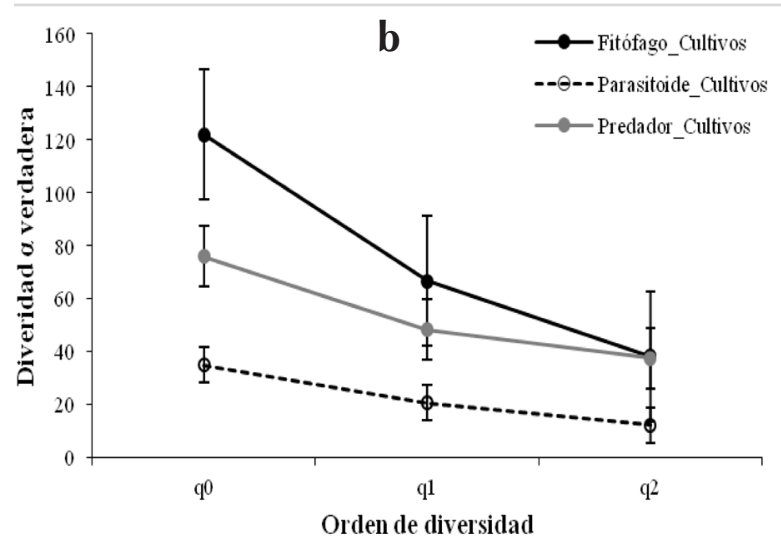

especies entre los dos tipos de cultivo, para la diversidad beta en términos generales fue alta ya que beta varió entre 1,1158 y 1,8462; la beta máxima que se podría obtener es de 2,0 (Figura 4a). La diversidad beta en términos del rol trófico fue alta para fitófago donde beta varió entre 1,1346 y 1,6446; mientras que para parasitoide y depredador fue baja donde beta varió entre 1,0505 - 1,1302 y 1,0063 - 1,0158; respectivamente (Figura 4b).

Al analizar la comunidad de artrópodos presentes para los bordes de cultivo maíz, arroz y algodón mediante las curvas de rango-abundancia en general se observa

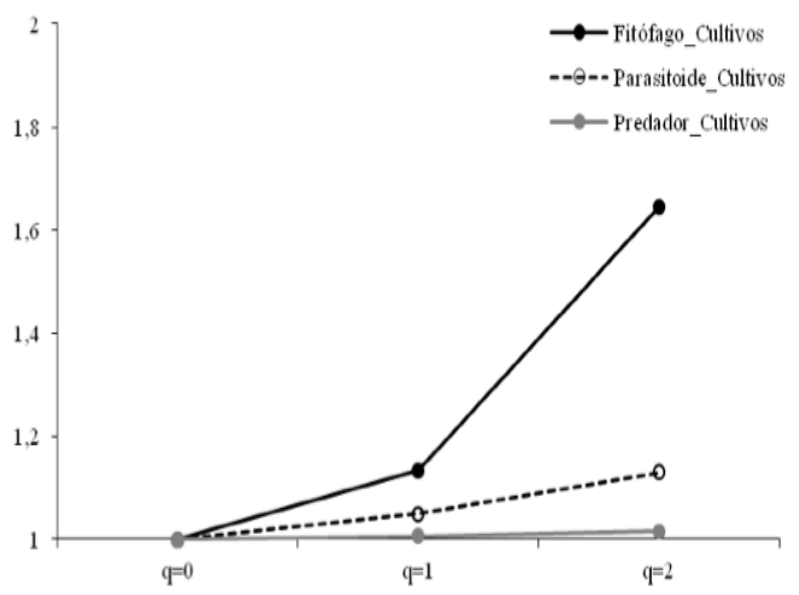

(a) para los artrópodos presen- 
que la pendiente de las gráficas de rangoabundancia indica una tendencia similar entre los bordes de los tipos de cultivo. Se registraron especies abundantes cada cultivo, para el cultivo de arroz, las especies Frankliniella occidentalis y Tagosodes orizicolus; para el cultivo de maíz, la especie F. occidentalis y la especie Solenopsis sp. y finalmente para el de algodón dominaron las especies Bemisia tabaci y Aphis gossypi, donde B. tabaci concentró el 43,11\% de los individuos colectados (Figura 5).

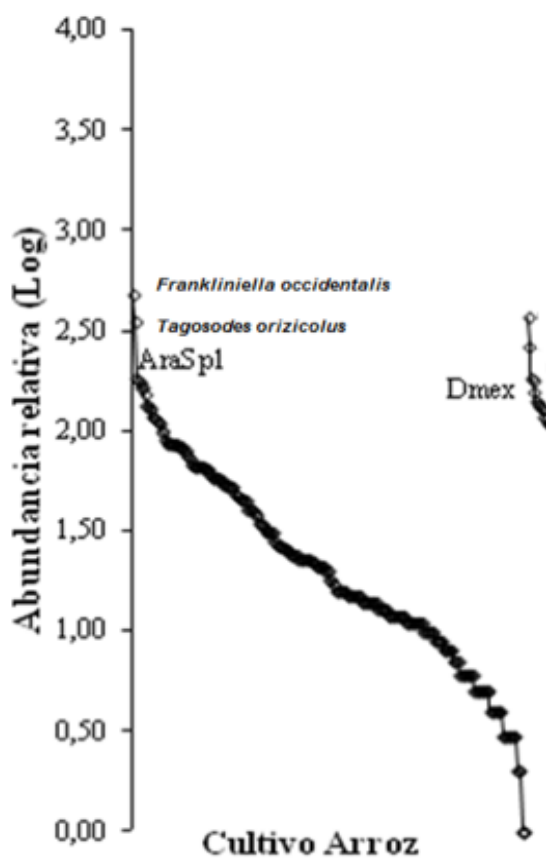

Figura 5. Curvas de rango tes en los bordes de cultivo
Cultivo Maiz

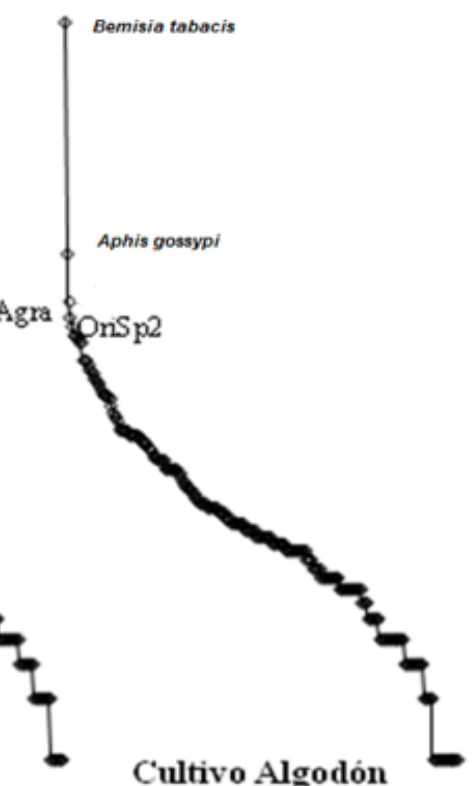

abundancia de artrópodos presenen Montería, Córdoba- Colombia..
También se registraron especies con menor abundancia consideradas dentro de un contexto ecológico como especies raras; las cuales dentro de los muestreos estuvieron representadas por un sólo individuo, para el borde del cultivo arroz la especie Pselliopus sp.; para el borde del cultivo maíz la especie Podisus sp. y para algodón las especies Tibraca limbativentris, Euschistus sp., Triatoma sp., Podisus sp. y la morfoespecie Corixidae sp1. Las curvas de rango-abundancia para los diferentes roles tróficos muestran que la pendiente de las gráficas indica una tendencia ligeramente diferente entre los diferentes roles tróficos. Los cultivo arroz y maíz presentaron dos grupos como codominantes fitófago y predador, mientras que para el cultivo algodón el rol fitófago mostró una clara dominancia con respecto a los demás (Figura 6).

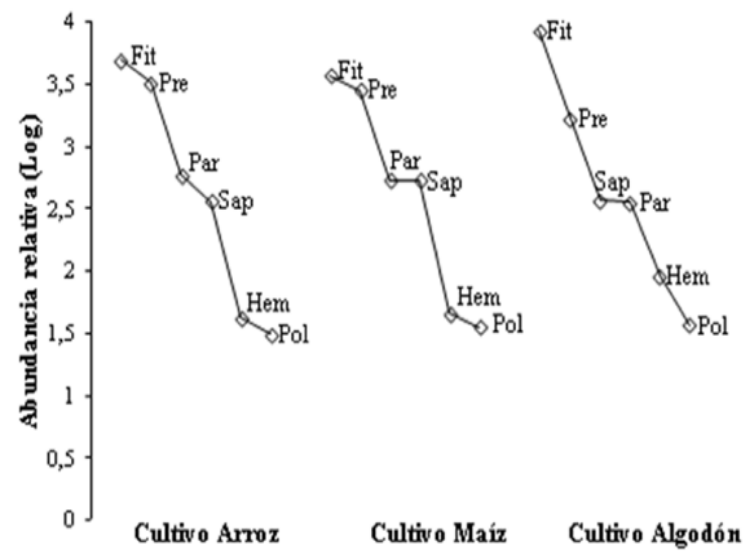

Figura 5. Curvas de rango abundancia de artrópodos presentes de acuerdo al rol funcional en bordes de cultivo en Montería, Córdoba- Colombia. 


\section{CONCLUSIONES}

Se reportaron 24 especies vegetales florecidas, correspondiente a 21 géneros de 16 familias botánicas de las cuales la familia Malvaceae y Fabaceae fueron las más diversas en los agroecosistemas maíz, arroz y algodón; quienes se ven beneficiados por la conservación de la biodiversidad silvestre y los arreglos de alta diversidad de insectos en los cultivos, favoreciendo la permanencia de los enemigos naturales como depredadores y parasitoides.

Las especies de arvenses que se identificaron en este estudio sirven como hábitats alternos para la hibernación y alimentación de la entomofauna asociada ya que requieren de polen o néctar, además que albergan presas para los enemigos naturales cubriendo épocas de ausencia de insectos indeseables.

A pesar de que los enemigos naturales varían en su respuesta a la distribución, densidad y dispersión en los cultivos de maíz, arroz y algodón, la evidencia señala que ciertos atributos estructurales de los diferentes agroecosistema, influyen significativamente en la dinámica y diversidad de la entomofauna asociada.

\section{REFERENCIAS}

Altieri, M y Nicholls, C. 2002. Biodiversidad y diseño agroecológico: un estudio de caso de manejo de plagas en viñedos. Manejo Integrado de Plagas y Agroecología (Costa Rica). 65: 50-64.

Altieri, M y Nicholls, C. 2012. Agroecología: única esperanza para la soberanía alimentaria y la resiliencia socioecológica. Artículo preparado para Conferencia de las Naciones Unidas sobre el Desarrollo Sostenible (Rio+20), con contribución de otros miembros de SOCLA. Río de Janeiro, Brasil.
Bailey L. y Bailey E. 1976. Hortus Third: A Concise Dictionary of Plants Cultivated in the United States and Canada. Macmillan Publishing Company N.Y , USA. 1 edition. 1290 pp.

Blanco, Y. y Leyva, A. 2007. Las arvenses en el agroecosistema y sus beneficios agroecológicos como hospederas de enemigos naturales. Cultivos tropicales. 28 (2):21-28.

Bennett, AF., Radford, JQ. y Haslem, A. 2006. Properties of land mosaics: Implications for nature conservation in agricultural environments. Biological

133(2):250-264.

Conservation org/10.1016/j.biocon.2006.06.008

Blanco, Y. y Leyva, A. 2009. Las arvenses y su entomofauna asociada en el cultivo del maíz (Zea mays, L.) posterior al periodo crítico de competencia. Cultivos tropicales. 30(1):11-17.

Blanco, Y. y Leyva, A. 2010. Abundancia y diversidad de especies de arvenses en el cultivo del maíz (Zea mays, L.) precedido de un barbecho transitorio después de la papa. Cultivos tropicales. 30(1): 11-17

Borror, D. y White, R. 1998. A field guide to insects: America north of Mexico (Vol. 19). Houghton Mifflin Harcourt.

Byrd, A. 1978. Tropica: Color Cyclopedia of Exotic Plants and Trees from the Tropics and Subtropics. Second Edition. Roehrs Company-Publishers. N.J, USA. ISBN 10: 0911266143 ISBN 13: 9780911266146.1136 pp.

Chao, C. y Jost, L. 2012. Coveragebased rarefaction and extrapolation: standardizing samples by completeness rather than size. Ecology, 93(12):2533-2547. https://doi.org/10.1890/11-1952.1

Denys, C. y Tscharntke, T. 2002. Plantinsect communities and predatorprey ratios in field margin strips, adjacent crop fields, and fallows. Oecologia 130(2):315-324. https:// doi.org/10.1007/s004420100796 
Gamboa, L. y Criollo, M. 2011. Forestería análoga y su rol en la recuperación de ecosistemas y el cambio climático. LESIA revista de agroecología. 27 (2): 8-12.

Faye P., Planchuelo A. y Molinelli M. 2002. Relevamiento de la Flora Apícola e Identificación de Cargas de Polen en el sureste de la provincia de Córdoba, Argentina. Agriscientia, VOL. XIX : 19-30.

Faye P. y Molinelli M. 2004. Cargas de Polen Provenientes del Sureste de la Provincia de Córdoba, Argentina. Espacio Apícola. 72 pp.

Feinsinger, P. 2003. El diseño de estudios de campo para la conservación de la biodiversidad. FAN (Fundación Amigos de la Naturaleza), Santa Cruz de la Sierra, Bolivia. 242 pp.

Fernández, F. and Sharkey M. (eds.) 2006. Introducción a los Hymenoptera de la Región Neotropical. Sociedad Colombiana de Entomología y Universidad Nacional de Colombia, Bogotá D. C., 894 pp.

Jost, L. 2006. Entropy and diversity. Oikos, 113:363-375. https://doi.org/10.1111/ j. $2006.0030-1299.14714$.x

Jost, L. 2007. Partitioning diversity into independent alpha and beta components. Ecology, 88: 2427-2439. https://doi.org/10.1890/06-1736.1

Landis, D; Wratten, S; Gurr, G. 2000. Habitat management to conserve natural enemies of arthropod pests in agriculture. Annual Review of Entomology 45:175-201. https://doi. org/10.1146/annurev.ento.45.1.175

Liogier, A. 1982. La Flora de la Española I. Universidad Central del Este (UCE) Vol. VI Serie Científica XII. Santo Domingo, República Dominicana; Editora Taller C. por A. 317 pp.

Liogier, A. 1983. La Flora de la Española II. Universidad Central del Este (UCE Vol. 44 Serie Científica XV. Santo Domingo, República Dominicana; Editora Taller C. por A. 420 pp.
Liogier, A. 1985. La Flora de la Española III. Universidad Central del Este (UCE) 39 Vol. LVI Serie Científica 22. Santo Domingo, República Dominicana; Editora Taller C. por A. 431 pp.

Liogier, A. 1986. La Flora de la Española IV. Universidad Central del Este (UCE) Vol. LXIV Serie Científica 24. Santo Domingo, República Dominicana; Editora Taller C. por A. 377 pp.

Liogier, A. 1989. La Flora de la Española V. Universidad Central del Este (UCE) Vol. LXIX Serie Científica 26. Santo Domingo, República Dominicana; Editora Taller C. por A. 398 pp.

Liogier, A. 1994. La Flora de la Española VI. Universidad Central del Este (UCE) Vol. LXX Serie Científica 27. Santo Domingo, República Dominicana; Editora Taller C. por A. 517 pp.

Liogier, A. 1995. La Flora de la Española VII. Universidad Central del Este (UCE) Vol. LXXI Serie Científica 28. Santo Domingo, República Dominicana; Editora Taller C. por A. 491 pp.

Liogier, A. 1996. La Flora de la Española VIII. Universidad Central del Este (UCE) Vol. LXXII Serie Científica 29. Santo Domingo, República Dominicana; Editora Taller C. por A. 588 pp.

Liogier, A. 2000. La Flora de la Española IX. Jardín Botánico Nacional "Dr. Rafael Ma. Moscoso", Instituto Tecnológico de Santo Domingo (INTEC). Santo Domingo, República Dominicana. Editora Corripio C. por A. 151 pp.

Magurran, A. 2004. Measuring Biological Diversity. Oxford, Blackwell Science Ltd., 256 pp.

Marshall, E.y Moonen,A.2002. Fieldmargins in northern Europe: their functions and interactions with agriculture. Agriculture, Ecosystems and Environment 89(1-2):5-21. https://doi. org/10.1016/S0167-8809(01)00315-2

McAlpine, J., Peterson, B., Shewell, G., Teskey, H., Vockeroth, J. and Wood, D. 1981. Manual of Nearctic Diptera. Volume 1 (No. 27). 
McGavin, G. 2001. Essential entomology: an order-by-order introduction. Oxford: Oxford University Press; Q (83)2.

McGavin, G. 2000. Insects, spiders and other terrestrial arthropods. London: Doring Kindersley; Q (84).

Mexzón, R. and Chinchilla, C. 2003. Especies vegetales atrayentes de la entomofauna benéfica en plantaciones de palma de aceite (Elaeis guineensis Jacq.) en Costa Rica. Revista Palmas, 24(1), 33-57.

Moreno, C., Barragan, F., Pineda, E. and Pavon, N. P. 2011. Reanalyzing alpha diversity:alternativestounderstandand compare information about ecological communities. Revista Mexicana de Biodiversidad, 82(4), 1249-1261.

Nicholls, C., Parrella, M. and Altieri, M. 2001. Effects of a vegetational corridor on the abundance and dispersal of insect biodiversity within a northern Californian organic vineyard. Landscape Ecology 16(2):133-146. https://doi. org/10.1023/A:1011128222867

Nicholls, C. 2004. Bases agroecológicas para diseñar e implementar una estrategia de manejo de hábitat para control biológico de plagas. Department of Environmental Science, Policy and Management, University of California, Berkeley. 24 pp.

Nicholls, C. 2006. Bases agroecológicas para diseñar e implementar una estrategia de manejo de hábitat para control biológico de plagas. Agroecología. 1: 37- 48.
Nicholls, C. 2008. Control Bilógico de insectos: un enfoque agroecológico. Medellín: Editorial Universidad de Antioquia.

Norris, R. y Kogan, M. 2005. Ecology of interactions between weeds and arthropods. Annual review of entomology 50:479-503. https://doi.org/10.1146/annurev. ento. 49.061802 .123218

Paleologos, M., Flores, C., Sarandon, S., Stupino, S. And Bonicatto, M. 2008. Abundancia y diversidad de la entomofauna asociada a ambientes semi-naturales en fincas hortícolas de La Plata, Buenos Aires, Argentina. Revista Brasileira deagroecología, 3(1).

Pérez G., Villarreal P. and Fernández, H. 2011. Bioecología del picudó del tallo del maiz Linogeraeus capillatus (Leconte) (Coleoptera: Curculionidae) en el departamento de Córdoba-Colombia. Revista Temas Agrarios. Vol. $16 \mathrm{~N}^{\circ} 1.23$ pp.

Rodríguez, M. y Van Hoof, B. 2003. Environmental performance of the Colombian oil palm industry. Colombia, Fedepalma (No. L-0564), 158 pp.

Saíni, E. and Polack, A. 2002. Enemigos naturales de los Trips sobre flores de malezas. RIA, 29 (1): 117 A 123 ISSN 0325-8718 INTA, Argentina RIA, 29 (1): 117 a 123. INTA.

Triplehorn, C. y Johnson, N. 2011. Estudo dos insetos - traducão da $7^{a}$ edição de borror and delong's introduction to the study of insects. São Paulo, Cengage Learning, 809 pp. 